\title{
Primary and Secondary Effects of Statin on Cerebro- Cardiovascular Disease Using the Nationwide Health Screening Data
}

\section{Jong Weon Lee}

National Health Insurance Service Ilsan Hospital

Dougho Park

Pohang Stroke and Spine Hospital

Han Eol Cho

Gangnam Severance Hospital

Jong Hun Kim

National Health Insurance Service Ilsan Hospital

Hyoung Seop Kim ( $\nabla$ rehappydoc@gmail.com )

National Health Insurance Service Ilsan Hospital

\section{Research Article}

Keywords: Cerebro-cardiovascular disease, Myocardial infarction, Stroke, Statins

Posted Date: August 10th, 2021

DOI: https://doi.org/10.21203/rs.3.rs-779664/v1

License: (c) (1) This work is licensed under a Creative Commons Attribution 4.0 International License. Read Full License 


\section{Abstract}

Background and Purpose Dyslipidemia is a significant risk factor for cerebro-cardiovascular diseases (CVD). Limited evidence is available on the prevention effect of statin in a nationwide large population. We aim to verify the primary and secondary prevention effects of statin on CVD among not only the general adult population but also elderly over 60 years.

Methods This study is a big data cohort study using propensity score-matched data from the Korean National Health Screening Cohort. Differences in the cumulative incidence of major adverse cerebrocardiovascular events and hazard ratio between the statin-user and the non-statin-user groups were investigated.

Results A propensity score-matched pairs of statin-user and non-statin-user identified 64,182 population of 40-75 years old without CVD and 24,688 with accompanying diseases. In this group, statin showed both primary (HR 0.76, 95\% $\mathrm{Cl} 0.70-0.83, \mathrm{p}<0.001)$ and secondary ( $\mathrm{HR} 0.87,95 \% \mathrm{Cl} 0.79-0.95, \mathrm{p}=0.004)$ prevention effect. In the elderly over 60 years, statin only showed primary prevention effect (HR $0.85,95 \%$ $\mathrm{Cl} 0.76-0.96, \mathrm{p}=0.008)$ in the 26,836 propensity score-matched pairs.

Conclusions Statin use in the elderly showed the primary prevention effect on CVD. Taking statin is desirable for both general population of dyslipidemia patients and elders over 60 years with CVD risk factors.

\section{Introduction}

In order to better prevent cerebro-cardiovascular diseases (CVDs), the 2013 and 2018 American College of Cardiology and the American Heart Association (ACC-AHA) guidelines called for increased statin use compared to the Third Adult Treatment Panel (ATP-III) guidelines of the National Cholesterol Education Program. ${ }^{1-3}$ The 2013 ACC-AHA guidelines recommended statin by implementing the risk threshold of atherosclerotic cardiovascular disease (ASCVD) and eliminating the low-density lipoprotein (LDL)cholesterol treatment goals. ${ }^{4}$ Even though some concerns were raised that the ACC-AHA guidelines substantially increased the statin eligibility ${ }^{5}$, the efficacy of the ACC-AHA guidelines has been verified by many studies involving diverse ethnicities. ${ }^{4-8}$

Even though advanced age is one of the significant risk factors for ASCVD ${ }^{9}$, less stringent treatment goals in elderly individuals with other cardiovascular risk factors, such as diabetes, have been in practice. ${ }^{10-12}$ Guidelines for LDL-cholesterol management, specifically in the elderly, have not been clear. While aspirin and clopidogrel, two important drugs in preventing CVDs, showed only secondary preventive effects, statin showed both primary and secondary prevention. ${ }^{13-16}$ However, previous studies have rarely focused strictly on the elderly and usually included mixed elderly and non-elderly populations. ${ }^{17-20}$ 
In our previous study with the National Health Insurance Service Health Screening Cohort (NHIS-HEALS), we reassessed the inclusion criteria of both the 2013 ACC-AHA and the ATP-III guidelines and verified the clinical effectiveness of the ACC-AHA guidelines in predicting the cumulative incidence of major adverse cerebro-cardiovascular events (MACE). ${ }^{20}$ Furthermore, the ACC-AHA guidelines increased statin eligibility mostly in the elder groups over 60 years old. Therefore, we aimed to analyze the efficacy of the 2013 ACCAHA guidelines not only in the general adult population but specifically among elderly individuals over 60 years regarding the primary and secondary prevention effects on CVD by reassessing the secondary analysis of our previous study.

\section{Methods}

\section{Study population}

In Korea, all employees must undergo a mandatory health screening examination annually, whereas the unemployed population is screened biannually. NHIS-HEALS gathered health screening data from January 2002 to December $2013 .^{21}$ Among the 5.15 million screened examinees aged $40-79$ years, as of December 2002, 10\% were randomly sampled $(n=514,866)$ and provided as cohort data to support academic research. Baseline characteristics, such as sex, age, body mass index (BMI), accompanying medical diseases, and other factors, were surveyed and followed. Since low-density lipoprotein cholesterol was first collected in 2009, the mean follow-up duration until 2013 was 4.6 years, and the cohort database was released in 2015.

We separated the available data first into adults 40-75 years with a background of MACE and further narrowed it down to elderly people 60-75 years old (eFigure 1). A total of 355,240 adults 40-75 years old and 122,398 elderly individuals 60-75 years old were studied in this research to find the cumulative incidence of MACE. We excluded participants over 75 years since this group would have been above the average life span during the follow-up period and could have confounded the mortality rate.

\section{Main outcome measures}

The primary outcomes included MACE, CVD mortality, fatal or nonfatal myocardial infarction (MI), and stroke. According to the International Statistical Classification of Diseases (ICD), CVD mortality was defined by the death codes with I20 125; MI with I21 classification; and stroke with I60 164 and I69 classifications. A MACE incident was added to the dataset when a patient was admitted to a hospital with a primary or secondary diagnosis code of the above diseases.

\section{Statistical analysis}

The cumulative incidence rates of MACE were plotted on the Kaplan-Meier survival curves. Participants were subdivided into the statin-user group and the non-statin-user group. For the statin-user group, the monitoring period started from statin administration and lasted until the MACE occurrence or the end of 
the follow-up duration. For the non-statin-user group, the monitoring period ranged from health screening examination to the date of MACE occurrence or the end of the follow-up duration.

The two groups were matched using the propensity score matching method for sex, age, BMI, waist circumference, hypertension, diabetes, triglyceride, high-density lipoprotein (HDL)-cholesterol, hemoglobin, blood pressure, and smoking status controlled. Diabetes was defined when a patient was diagnosed with ICD code for diabetes, treated with medication for diabetes, or showed fasting glucose higher than $126 \mathrm{mg} / \mathrm{dL}$ in health screening test. Hypertension was defined when a patient was diagnosed with ICD code for hypertension, treated with antihypertensive medication, showed systolic blood pressure higher than $140 \mathrm{mmHg}$ or diastolic blood pressure higher than $90 \mathrm{mmHg}$ in health screening test. In the patient group with current ASCVD and those without ASCVD, the cumulative incidence rates of MACE were compared between the statin-user group and the non-statin-user group.

All analyses in this study were performed using the SAS software (version 9.4, SAS Institute, Cary, NC, USA) and R version 4.1 (R Foundation for Statistical Computing, Vienna, Austria). An alpha level of $<0.05$ and non-overlapping $95 \%$ confidence intervals (Cls) were used as the criteria for statistical significance in all tests.

The study was conducted according to the guidelines of the Declaration of Helsinki. The need for patient consent was waived due to the use of a cohort big data, and all components and procedures of this study were approved by the Institutional Review Board of the National Health Insurance Service Ilsan Hospital (IRB file No. 2020-02-001) and the NHIS data sharing system (Approval No. NHIS-2021-2-129).

\section{Results}

\section{Demographic data}

A total of 355,240 participants were $40-75$ years old; 122,398 were age $60-75$ years. Among the $40-75$ year-old group, 32,091 participants without current ASCVD used statin for primary prevention, and 12,344 patients with current ASCVD used statin for secondary prevention. Among the 60-75 year-old elderly group, 13,418 participants used statin for primary prevention, whereas 6,539 patients used statin for secondary prevention. The demographic data are shown in Table 1.

For both groups with and without current ASCVD, the propensity score matching analysis was used to design a non-treated control group. After propensity score matching, sex, age, BMI, waist circumference, hypertension, diabetes, triglyceride, high-density lipoprotein cholesterol, hemoglobin, blood pressure, and smoking status did not show statistically significant differences between the two groups. (eTables 1 \& 2 ) However, the total cholesterol level between the statin-user and the non-statin-user elderly with current ASCVD was statistically different (eTable 2).

\section{Cumulative incidence of MACE in patients without current ASCVD (Primary prevention)}


Figure 1 depicts the Kaplan Meier survival curve, showing the cumulative incidence of MACE in 40-75year-old participants without current ASCVD. Figure 2 shows the cumulative incidence of MACE in 60-75year-old participants. During the mean follow-up duration of 4.6 years, the statin-user group showed significantly lower multivariable hazard ratios of MACE in both age groups (HR $0.76,95 \% \mathrm{Cl}=0.70-0.83$, $p<0.001$ for the $40-75$ age group \& HR $0.85,95 \% \mathrm{Cl}=0.76-0.96, p=0.008$ for the $60-75$ age group), demonstrating the statistically significant primary prevention effect of statin.

\section{Cumulative incidence of MACE in patients with current ASCVD (Secondary prevention)}

Figure 3 depicts the Kaplan Meier survival curve showing the cumulative incidence of MACE in the 4075-year-old patients with current ASCVD. During the follow-up duration, the statin-user group showed a significantly lower multivariable hazard ratio of MACE (HR 0.87, 95\% $\mathrm{Cl}=0.79-0.95, \mathrm{p}=0.004)$, demonstrating the statistically significant secondary prevention effect of statin among the general adult population.

The cumulative incidence of MACE in the 60-75-year-old patients with current ASCVD is shown on the Kaplan Meier survival curve of Figure 4. The multivariable hazard ratio of MACE in the statin-user group was not statistically significant (HR $0.92,95 \% \mathrm{Cl}=0.81-1.03, \mathrm{p}=0.182)$.

\section{Discussion}

In the present study, statin showed primary and secondary preventive effects on cerebro-cardiovascular diseases in the 40-75-year-old adult group. However, only the primary preventive effect was statistically significant in the 60-75-year-old elderly group. Our findings elucidate that strict LDL-cholesterol control in elderly individuals aged $60-75$ years is beneficial for the primary prevention of MACE.

The primary and secondary preventive effects of statin have been proven in previous studies. For primary prevention, Downs et al. showed a reduced risk for the first acute major coronary events in men and women aged 45-73 years, with average values for total cholesterol and LDL-cholesterol. ${ }^{22} \mathrm{Kim}$ et al. specifically recruited 1,278 elderly individuals over 75 years to validate the effect of statin. ${ }^{23}$ Their study proved lower cardiovascular events and all-cause deaths with statin use, but the study was limited to participants from a single tertiary hospital. Kim et al. additionally used the National Health Insurance Services-Senior Cohort of 1,370 elderly patients over 75 years to demonstrate the primary prevention effect of statin in decreasing the all-cause mortality if statin was used for at least 5 years. ${ }^{24}$ Our study similarly showed the primary preventive effect of statin. However, it differed in that we used the nationwide health screening cohort data, which included a broader age range and a greater number of elderly individuals.

The Heart Protection Study (HPS) ${ }^{25}$, Pravastatin or Atorvastatin Evaluation and Infection TherapyThrombolysis in Myocardial Infarction 22 trial (PROVE-IT) ${ }^{26}$ and Reversal of Atherosclerosis with 
Aggressive Lipid Lowering studies ${ }^{27}$, all demonstrated the secondary effect of statin in preventing MACE among patients with accompanying coronary diseases, occlusive arterial diseases, or diabetes.

Another important drugs to prevent cardiovascular events are aspirin and clopidogrel. In secondary prevention trials of aspirin, the potential benefits of reducing the occurrence of MACE were significant and outweighed the potential risk of hemorrhages. ${ }^{13-15}$ In primary prevention trials, however, aspirin did not show an effect on MACE. In the Aspirin to Reduce Risk of Initial Vascular Events trial ${ }^{28}$, aspirin showed no effect on MACE in adults with multiple risk factors for cardiovascular diseases but showed increased gastrointestinal bleeding. In the Aspirin in Reducing Events in the Elderly trial ${ }^{29}$, aspirin was randomly assigned to the patient group who were 70 years or older, but the study was terminated early for futility. Aspirin had no effect on disability-free survival but significantly increased the risk of major hemorrhage and all-cause mortality.

Similar to aspirin, the secondary preventive effect of clopidogrel is proved through multiple studies. Bhatt et al. showed lower MACE with clopidogrel compared to the placebo group. ${ }^{16}$ Moreover, this benefit was more pronounced in patients with prior MI or ischemic stroke than peripheral artery disease. In the Clopidogrel for High Atherothrombotic Risk and Ischemic Stabilization, Management, and Avoidance (CHARISMA) study ${ }^{30}$, however, the primary preventive effect of MACE by clopidogrel was not statistically significant; what is more, a higher risk of severe bleeding was seen in the primary prevention group compared with the secondary prevention group. Currently, no studies have demonstrated the primary preventive effect of aspirin and clopidogrel on CVDs.

In comparison to aspirin and clopidogrel, statin showed both primary and secondary preventive effects on CVDs. Moreover, continued lowering of LDL-cholesterol through statin use is recommended in the elder group over 60 years old. Sabatine et al. even suggested that further lowering of LDL-cholesterol can further reduce cardiovascular risks. ${ }^{31}$ Other studies, such as HPS and PROVE-IT, suggested continued lowering of LDL-cholesterol to levels substantially below current guideline recommendations, regardless of the initial LDL-cholesterol concentrations. ${ }^{25-26}$

We focused on the elder group due to the less stringent treatment goals in elderly individuals with other cardiovascular risk factors, such as hypertension and diabetes. Until Beckett et al. published the Hypertension in the Very Elderly Trial $^{32}$, epidemiologic studies pointed to the inverse relationship between blood pressure and the risk of death among people over 80 years old, thus possibly reflecting the increased risks of strict blood pressure control. For diabetes control, hemoglobin A1c, blood glucose levels, cognitive function, life expectancy, functional impairments, polypharmacy, nutritional defects, and other factors for overall health care must be all considered for the diabetic elderly people over 65 years old. ${ }^{10-12}$ Since the timeline of benefit is estimated to be at least 8 years for glycemic control ${ }^{11}$, the decision to treat diabetes should be based on the benefit and risk ratio for everyone concerned. Gerstein et al. even demonstrated excessive deaths with intensive glucose control in the elderly while MACE was not significantly reduced. ${ }^{33}$ 
Previous studies using the 2013 ACC-AHA guidelines showed an improved CVD prediction and called for increased statin eligibility among elderly and diabetic patients. ${ }^{8,20,34}$ Baigent et al. conducted a metaanalysis showing further LDL-cholesterol reduction has a definite effect of reducing the incidence of heart attack and ischemic stroke. ${ }^{35}$ Therefore, our study investigated and proved the primary and secondary effects of statin in preventing cardiovascular diseases among adults from not only the total population of 40-75 year-olds but also the elderly group of $60-75$ year-olds. Since a set LDL-cholesterol threshold is controversial and asymptomatic atherosclerosis begins during childhood ${ }^{36}$, future studies are needed to consider early statin use or to recommend strict LDL-cholesterol reference when a patient has risk factors for CVDs.

This study was subject to a few limitations. Administration of drugs that may affect the incidence of MACE, such as aspirin and clopidogrel, has not been investigated. Since antiplatelets and anticoagulants can function as confounding variables, it is necessary to control medications in the study. Furthermore, the mean follow-up duration was limited to 4.6 years due to LDL-cholesterol data availability. Longer follow up period is critical to determine the severity of MACE.

\section{Conclusions}

From the results of our study and previous studies, we confirm that statin has both primary and secondary prevention effects. When limiting the study sample to elderly individuals of 60-75 years old, the primary preventive effect was also demonstrated. Therefore, the 2013 ACC-AHA guidelines hold value in the elderly individuals over 60 years old when compared to the 2008 guidelines. Finally, the following study is essential to set the strict LDL-cholesterol reference for preventing MACE.

\section{Declarations}

\section{Acknowledgments}

This study was supported by the National Health Insurance Ilsan Hospital research fund.

\section{Competing Interests}

All authors declare no competing interests.

\section{Author contributions}

Conceptualization: JWL, JHK, HSK

Data curation: JWL, HSK

Formal analysis: JWL, HSK

Methodology: DP, HEC, HSK 
Supervision: DP, HEC, JHK, HSK

Validation: JHK, HSK

Writing-original draft: JWL

Writing-review\&editing: JWL, JHK, HSK

\section{Data Availability}

The datasets generated during and/or analysed during the current study are available from the corresponding author on reasonable request.

\section{Sources of Funding}

This study was supported by National Health Insurance Ilsan Hospital research fund.

\section{Conflicts of Interest \& Disclosures}

All authors have no conflicts of interest to declare.

\section{References}

1. National Cholesterol Education Program (NCEP) Expert Panel on Detection, Evaluation, and Treatment of High Blood Cholesterol in Adults (Adult Treatment Panel III). Third report of the National Cholesterol Education Program (NCEP) Expert Panel on detection, evaluation, and treatment of high blood cholesterol in adults (Adult Treatment Panel III) final report. Circulation 106(25), 3143-3421 (2002).

2. Goff, D.C., Jr., et al. 2013 ACC/AHA guideline on the assessment of cardiovascular risk: a report of the American College of Cardiology/American Heart Association Task Force on Practice Guidelines. J Am Coll Cardiol 63(25 Pt B), 2935-2959 (2014). doi:10.1016/j.jacc.2013.11.005

3. Grundy, S.M. et al. 2018 AHA/ACC/AACVPR/AAPA/ABC/ACPM/ADA/AGS/APhA/ASPC/NLA/PCNA guideline on the management of blood cholesterol: a report of the American College of Cardiology/American Heart Association Task Force on Clinical Practice Guidelines. Circulation 139(25), e1082-e1143 (2019). doi:10.1161/cir.0000000000000625

4. Stone, N.J., et al. 2013 ACC/AHA guideline on the treatment of blood cholesterol to reduce atherosclerotic cardiovascular risk in adults: a report of the American College of Cardiology/American Heart Association Task Force on Practice Guidelines. Circulation 129(25 Suppl 2), S1-45 (2014). doi:10.1161/01.cir.0000437738.63853.7a

5. Kavousi, M., et al. Comparison of application of the ACC/AHA guidelines, Adult Treatment Panel III guidelines, and European Society of Cardiology guidelines for cardiovascular disease prevention in a European cohort. JAMA 311(14), 1416-1423 (2014). doi:10.1001/jama.2014.2632 
6. Arai, H., Sasaki, J., Teramoto, T. Comment on the new guidelines in USA by the JAS Guidelines Committee. J Atheroscler Thromb 21(2), 79-81 (2014). doi:10.5551/jat.ed001

7. Muntner, P., et al. Validation of the atherosclerotic cardiovascular disease Pooled Cohort risk equations. JAMA 311(14), 1406-1415 (2014). doi:10.1001/jama.2014.2630

8. DeFilippis, A.P., et al. An analysis of calibration and discrimination among multiple cardiovascular risk scores in a modern multiethnic cohort. Ann Intern Med 162(4), 266-275 (2015). doi:10.7326/m14-1281

9. Lewington, S., et al. Blood cholesterol and vascular mortality by age, sex, and blood pressure: a metaanalysis of individual data from 61 prospective studies with 55,000 vascular deaths. Lancet 370(9602), 1829-1839 (2007). doi:10.1016/s0140-6736(07)61778-4

10. Kirkman, M.S., et al. Diabetes in older adults. Diabetes Care 35(12), 2650-2664 (2012). doi:10.2337/dc12-1801

11. Brown, A.F., Mangione, C.M., Saliba, D., Sarkisian, C.A. Guidelines for improving the care of the older person with diabetes mellitus. J Am Geriatr Soc 51(5 Suppl Guidelines), S265-280 (2003). doi:10.1046/j.1532-5415.51.5s.1.x

12. Sinclair, A.J., Paolisso, G., Castro, M., Bourdel-Marchasson, I., Gadsby, R., Rodriguez Mañas, L. European Diabetes Working Party for Older People 2011 clinical guidelines for type 2 diabetes mellitus. Executive summary. Diabetes Metab 37(Suppl 3), S27-38 (2011). doi:10.1016/s12623636(11)70962-4

13. Hennekens, C.H., Dyken, M.L., Fuster, V. Aspirin as a therapeutic agent in cardiovascular disease: a statement for healthcare professionals from the American Heart Association. Circulation 96(8), 27512753 (1997). doi:10.1161/01.cir.96.8.2751

14. Ajani, U.A., Ford, E.S., Greenland, K.J., Giles, W.H., Mokdad, A.H. Aspirin use among U.S. adults: Behavioral Risk Factor Surveillance System. Am J Prev Med 30(1), 74-77 (2006). doi:10.1016/j.amepre.2005.08.042

15. Ittaman, S.V., VanWormer, J.J., Rezkalla, S.H. The role of aspirin in the prevention of cardiovascular disease. Clin Med Res 12(3-4), 147-154 (2014). doi:10.3121/cmr.2013.1197

16. Bhatt, D.L., et al. Patients with prior myocardial infarction, stroke, or symptomatic peripheral arterial disease in the CHARISMA trial. J Am Coll Cardio/ 49(19), 1982-1988 (2007). doi:10.1016/j.jacc.2007.03.025

17. Ridker, P.M., et al. Rosuvastatin to prevent vascular events in men and women with elevated Creactive protein. N Engl J Med 359(21), 2195-2207 (2008). doi:10.1056/NEJMoa0807646

18. Nakamura, H., et al. Primary prevention of cardiovascular disease with pravastatin in Japan (MEGA Study): a prospective randomised controlled trial. Lancet 368(9542), 1155-1163 (2006). doi:10.1016/s0140-6736(06)69472-5

19. Yusuf, S., et al. Cholesterol lowering in intermediate-risk persons without cardiovascular disease. $N$ Engl J Med 374(21), 2021-2031 (2016). doi:10.1056/NEJMoa1600176 
20. Lee, J.W., Lim, H., Kim, J.H., Kim, H.S. Reassessment of inclusion criteria in the 2013 the American College of Cardiology and the American Heart Association Cholesterol Guidelines for Cardiovascular Disease Prevention. J Clin Neurol 17(1), 86-95 (2021). doi:10.3988/jcn.2021.17.1.86

21. Seong, S.C., et al. Cohort profile: the National Health Insurance Service-National Health Screening Cohort (NHIS-HEALS) in Korea. BMJ Open 7(9), e016640 (2017). doi:10.1136/bmjopen-2017-016640

22. Downs, J.R., et al. Primary prevention of acute coronary events with lovastatin in men and women with average cholesterol levels: results of AFCAPS/TexCAPS. Air Force/Texas Coronary Atherosclerosis Prevention Study. JAMA 279(20), 1615-1622 (1998). doi:10.1001/jama.279.20.1615

23. Kim, K., et al. Statin and clinical outcomes of primary prevention in individuals aged $>75$ years: The SCOPE-75 study. Atherosclerosis 284, 31-36 (2019). doi:10.1016/j.atherosclerosis.2019.02.02624.

24. Kim, S., Choi, H., Won, C.W. Effects of Statin Use for primary prevention among adults aged 75 years and older in the National Health Insurance Service Senior Cohort (2002-2015). Ann Geriatr Med Res 24(2), 91-98 (2020). doi:10.4235/agmr.20.0028

25. Heart Protection Study Collaborative Group. MRC/BHF Heart Protection Study of cholesterol lowering with simvastatin in 20,536 high-risk individuals: a randomised placebo-controlled trial. Lancet 360(9326), 7-22 (2002). doi:10.1016/s0140-6736(02)09327-3

26. Rouleau, J. Improved outcome after acute coronary syndromes with an intensive versus standard lipid-lowering regimen: results from the Pravastatin or Atorvastatin Evaluation and Infection TherapyThrombolysis in Myocardial Infarction 22 (PROVE IT-TIMI 22) trial. Am J Med 2005;118 Suppl 12A:28-35. doi:10.1016/j.amjmed.2005.09.014

27. Nissen SE, Tuzcu EM, Schoenhagen P, Brown BG, Ganz P, Vogel RA, et al. Effect of intensive compared with moderate lipid-lowering therapy on progression of coronary atherosclerosis: a randomized controlled trial. JAMA 2004;291(9):1071-1080. doi:10.1001/jama.291.9.1071

28. Gaziano JM, Brotons C, Coppolecchia R, Cricelli C, Darius H, Gorelick PB, et al. Use of aspirin to reduce risk of initial vascular events in patients at moderate risk of cardiovascular disease (ARRIVE): a randomised, double-blind, placebo-controlled trial. Lancet 2018;392(10152):1036-1046. doi:10.1016/s0140-6736(18)31924-x

29. McNeil JJ, Wolfe R, Woods RL, Tonkin AM, Donnan GA, Nelson MR, et al. Effect of aspirin on cardiovascular events and bleeding in the healthy dlderly. N Engl J Med 2018;379(16):1509-1518. doi:10.1056/NEJMoa1805819

30. Bhatt DL, Fox KA, Hacke W, Berger PB, Black HR, Boden WE, et al. Clopidogrel and aspirin versus aspirin alone for the prevention of atherothrombotic events. N Engl J Med 2006;354(16):1706-1717. doi:10.1056/NEJMoa06098932.

31. Sabatine MS, Wiviott SD, Im K, Murphy SA, Giugliano RP. Efficacy and safety of further lowering of low-density lipoprotein cholesterol in patients starting with very low levels: a meta-analysis. JAMA Cardiol 2018;3(9):823-828. doi:10.1001/jamacardio.2018.2258

32. Beckett NS, Peters R, Fletcher AE, Staessen JA, Liu L, Dumitrascu D, et al. Treatment of hypertension in patients 80 years of age or older. N Engl J Med 2008;358(18):1887-1898. 
doi:10.1056/NEJMoa0801369

33. Gerstein HC, Miller ME, Byington RP, Goff DC Jr., Bigger JT, Buse JB, et al. Effects of intensive glucose lowering in type 2 diabetes. N Engl J Med 2008;358(24):2545-2559. doi:10.1056/NEJMoa0802743

34. Ko MJ, Kim YJ, Park CM, Lee SM, Lee WJ, Pencina MJ, et al. Applicability and potential clinical effects of 2013 cholesterol guidelines on major cardiovascular events. Am Heart J 2015;170(3):598605.e597. doi:10.1016/j.ahj.2015.06.012

35. Baigent C, Blackwell L, Emberson J, Holland LE, Reith C, Bhala N, et al. Efficacy and safety of more intensive lowering of LDL cholesterol: a meta-analysis of data from 170,000 participants in 26 randomised trials. Lancet 2010;376(9753):1670-1681. doi:10.1016/s0140-6736(10)61350-5

36. Hong YM. Atherosclerotic cardiovascular disease beginning in childhood. Korean Circ J 2010;40(1):19. doi:10.4070/kcj.2010.40.1.1

\section{Tables}

Table 1. Demographic data of the study sample 


\begin{tabular}{|c|c|c|}
\hline & $\begin{array}{l}40-75 \text { years of age study } \\
\text { sample }\end{array}$ & $\begin{array}{l}60-75 \text { years of age study } \\
\text { sample }\end{array}$ \\
\hline Participants & 355,240 & 122,398 \\
\hline Statin-user, No. (\%) & 73,381 (20.7) & $33,267(27.2)$ \\
\hline Male, No. (\%) & $191,228(53.8)$ & $62,067(50.7)$ \\
\hline Age, mean (SD), years & $58.5(8.9)$ & $66.4(4.7)$ \\
\hline BMI, mean (SD), $\mathrm{kg} / \mathrm{m}^{2}$ & $24.0(2.9)$ & $24.1(3.0)$ \\
\hline Waist, mean (SD), cm & $82.0(8.3)$ & $83.1(8.1)$ \\
\hline \multicolumn{3}{|l|}{$\begin{array}{l}\text { Economic status ( } 10^{\text {th }} \text { percentiles), } \\
\text { No. }(\%)\end{array}$} \\
\hline 0 (Medical Aid) & $371(0.1)$ & $211(0.2)$ \\
\hline $1 \sim 3$ & $71,885(20.2)$ & $29,228(23.9)$ \\
\hline $4 \sim 6$ & $81,180(22.9)$ & $28,977(23.7)$ \\
\hline 7 9 & $130,958(36.9)$ & $43,911(35.9)$ \\
\hline 10 (highest) & $70,846(19.9)$ & $20,071(16.4)$ \\
\hline Hypertension, No. (\%) & $10,272(2.9)$ & $5,679(4.6)$ \\
\hline Diabetes, No. (\%) & $74,329(20.9)$ & $34,641(28.3)$ \\
\hline Total cholesterol, mean (SD), mg/dL & $200.1(37.7)$ & $198.3(38.6)$ \\
\hline Triglyceride, mean (SD) mg/dL & $140.3(91.6)$ & $140.2(85.9)$ \\
\hline $\mathrm{HDL}$, mean (SD), mg/dL & $55.3(30.9)$ & $54.7(31.7)$ \\
\hline LDL, mean (SD), mg/dL & $118.6(38.1)$ & $117.5(38.6)$ \\
\hline Hemoglobin, mean (SD), g/dL & $13.8(1.5)$ & $13.6(1.4)$ \\
\hline Systolic BP, mean (SD), mm Hg & $125.3(15.3)$ & $128.4(15.6)$ \\
\hline Diastolic BP, mean (SD), mm Hg & $77.6(10.0)$ & $78.0(9.8)$ \\
\hline Previous $\mathrm{CVD}^{\dagger}{ }^{\dagger}$, No. $(\%)$ & $35,016(9.9)$ & $17,696(14.5)$ \\
\hline \multicolumn{3}{|l|}{ Smoking status, No. (\%) } \\
\hline Non-smoking & $294,340(82.9)$ & $106,505(87.0)$ \\
\hline Current smoking & $60,900(17.1)$ & $15,893(13.0)$ \\
\hline Drinking, mean (SD), No./week & $1.9(1.6)$ & $1.8(1.7)$ \\
\hline
\end{tabular}


† Myocardial infarction, stroke, coronary artery disease

Abbreviations: BMI, Body mass index; HDL, High-density lipoprotein; LDL, Low-density lipoprotein; BP, Blood pressure; CVD, Cardiovascular disease

\section{Figures}

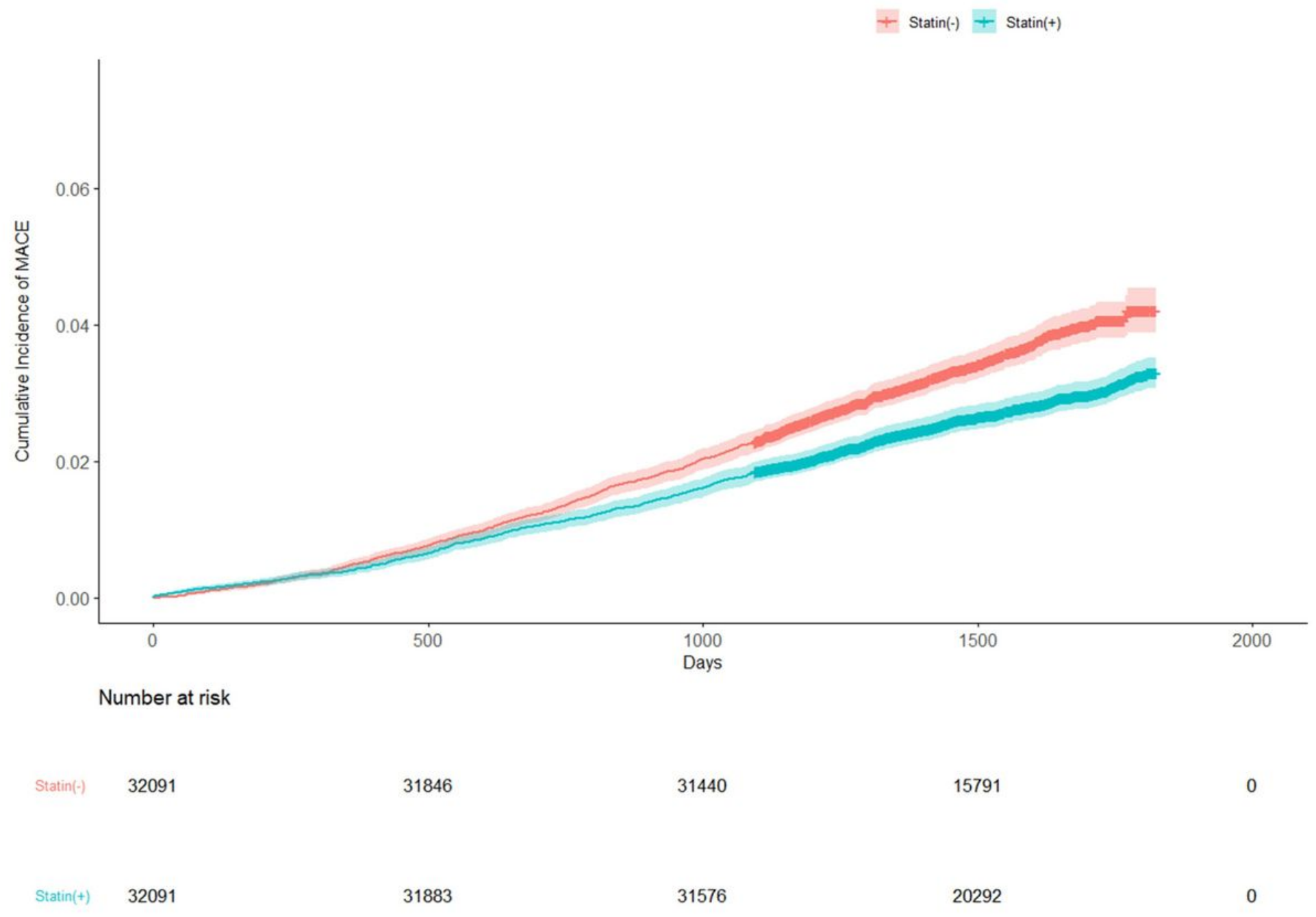

\section{Figure 1}

Kaplan-Meier survival curves for cumulative incidence of MACE in the 40-75 year-old participants without current ASCVD 


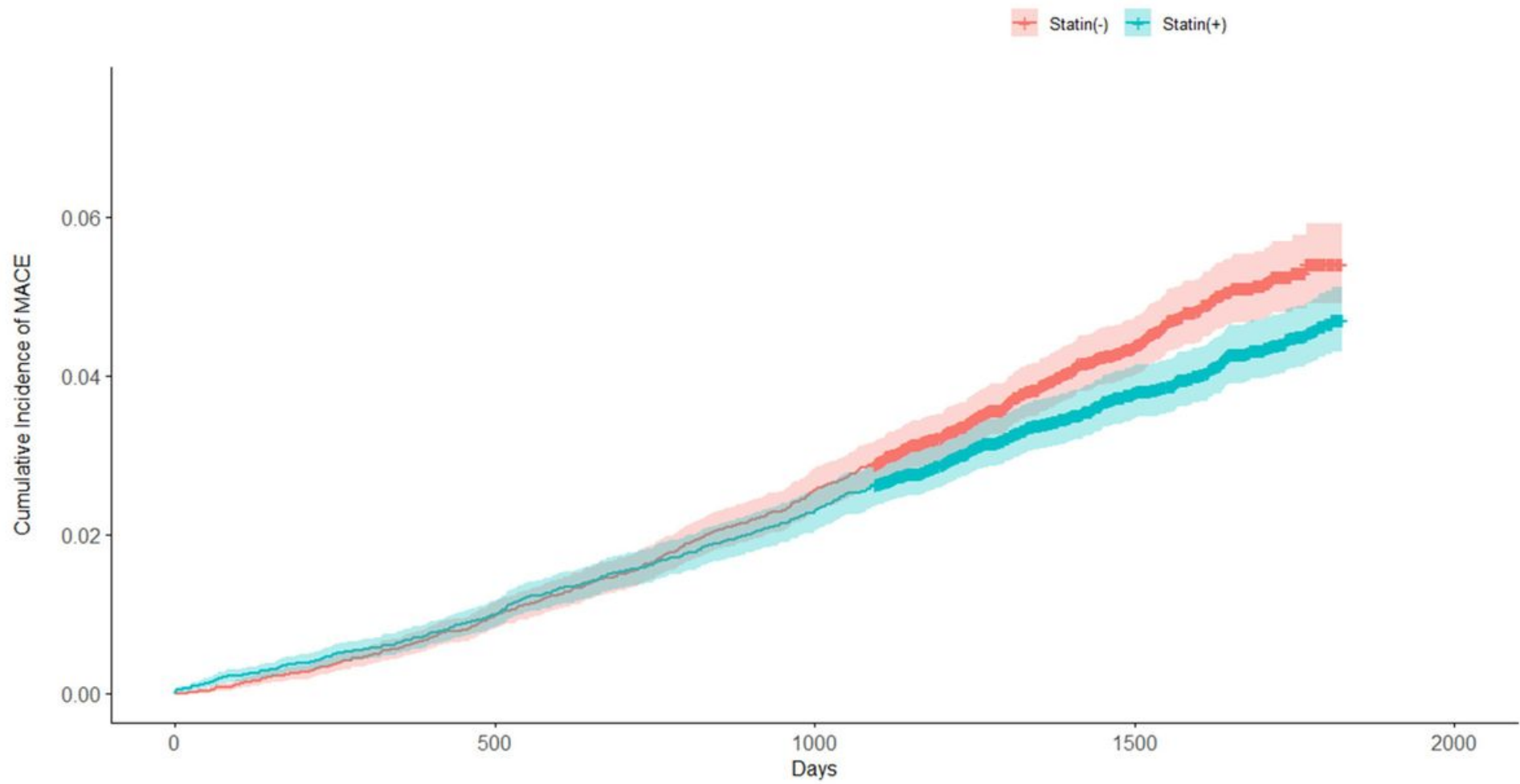

Number at risk

6534

0

Statin(+)

13418

13285

13111

8717

0

\section{Figure 2}

Kaplan-Meier survival curves for cumulative incidence of MACE in the 60-75 years-old participants without current ASCVD 


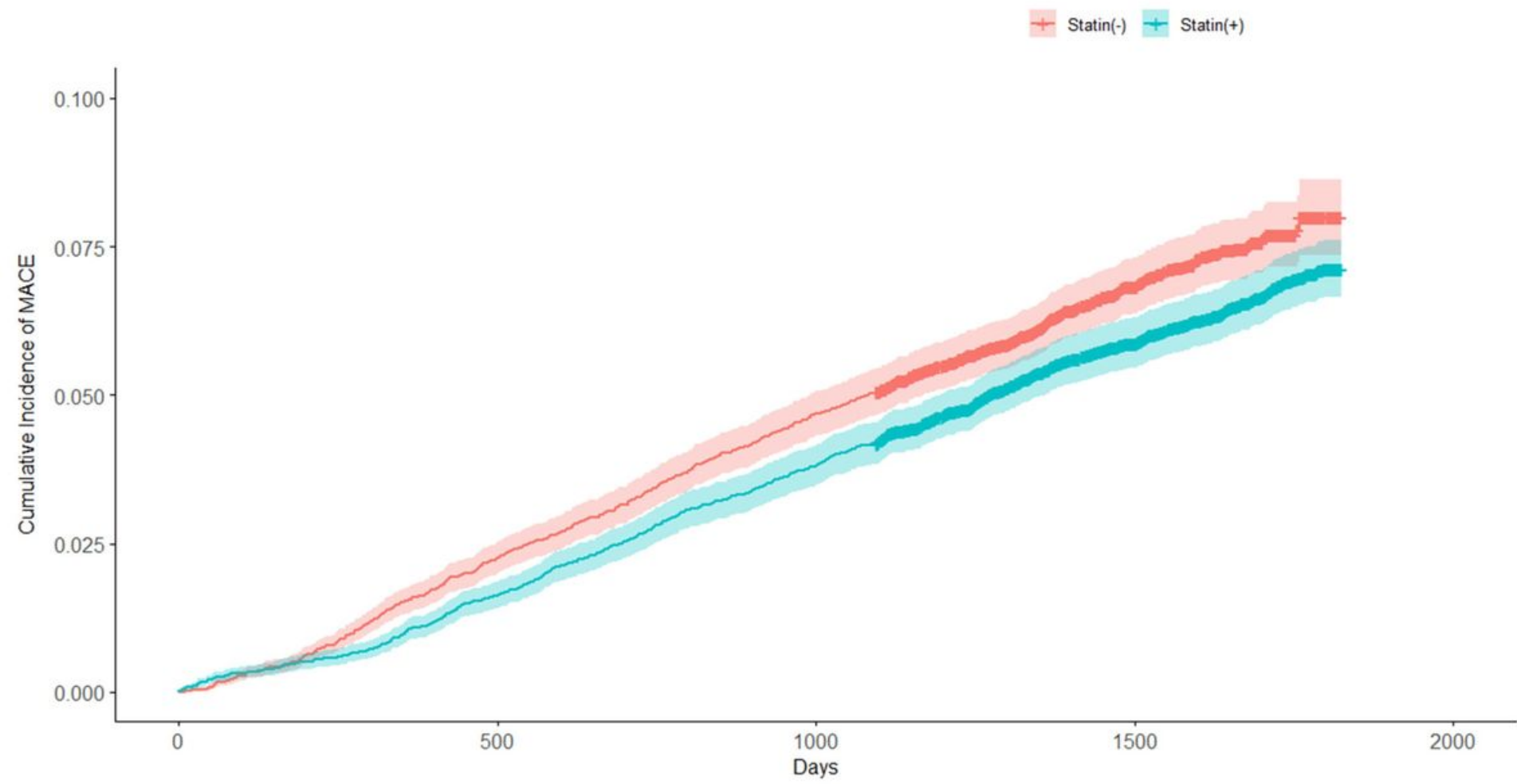

Number at risk

9571

\section{Figure 3}

Kaplan-Meier survival curves for cumulative incidence of MACE in the 40-75 year-old patients with current ASCVD 


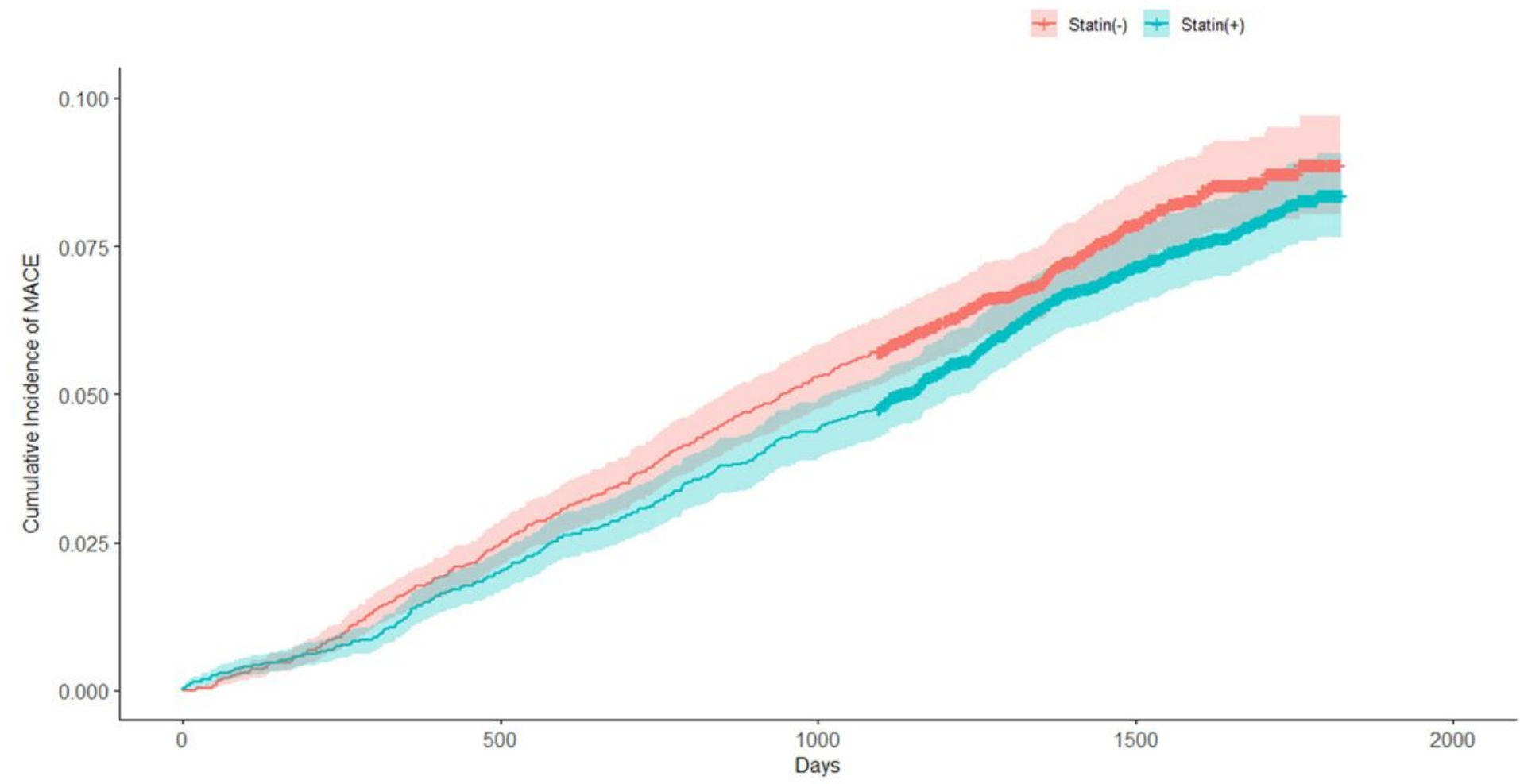

Number at risk

6193

\section{Figure 4}

Kaplan-Meier survival curves for cumulative incidence of MACE in the $60-75$ year-old patients with current ASCVD

\section{Supplementary Files}

This is a list of supplementary files associated with this preprint. Click to download.

- SCSupplementarydata.docx

- eFigure1.jpg 